\title{
The COVID-19 Pandemic and Rheumatology: Impact on Providing Care in Latin America and Around the World
}

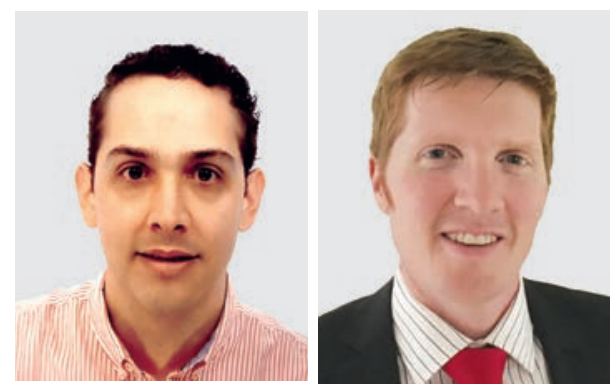

\author{
Sebastian E. Sattui ${ }^{1}$ (D) and Philip C. Robinson ${ }^{2}$ (D)
}

The novel coronavirus pandemic has affected the world, importantly, from a health perspective. Initial concern about all rheumatology patients being at risk has given way to a more nuanced view of the risks. ${ }^{1}$ The risk factors for poor outcomes are the same as in nonrheumatological patients, except some medications such as rituximab also increase risk. ${ }^{2}$ There are also potentially regional differences, as has been reported for Latin America. ${ }^{3}$ Now that vaccines are becoming available, we can start to see how a path back to "normal" might be achieved.

In the midst of all of this, however, are the regions still battling massive infection rates, such as India and South America. Globally, healthcare professionals and healthcare systems have been forced to rapidly adapt and respond to the demands of care. Physicians have been redeployed to the front lines of the coronavirus disease 2019 (COVID-19) pandemic, and healthcare systems have been redesigned to care for the rising numbers of patients. As a response to the surge of cases, and this is particularly true during the first waves of the pandemic, the COVID-19 focus of healthcare systems worldwide led to neglect of other ongoing needs of patients. ${ }^{4}$

In this issue of The Journal of Rheumatology, Fernández-Ávila and colleagues present the results of an observational

${ }^{1}$ S.E. Sattui, MD, MS, Assistant Professor of Medicine, Division of Rheumatology and Clinical Immunology, Department of Medicine, University of Pittsburgh School of Medicine, Pittsburgh, Pennsylvania, USA; ${ }^{2}$ P.C. Robinson, $M B C h B, P h D$, Associate Professor, University of Queensland School of Clinical Medicine, Faculty of Medicine, and Royal Brisbane \& Women's Hospital, Metro North Hospital \& Health Service, Herston, Queensland, Australia.

PCR reports personal fees from AbbVie, Atom Biosciences, Eli Lilly, Gilead, Janssen, Novartis, UCB, Roche, and Pfizer; meeting attendance support from BMS, Eli Lilly, Pfizer, and UCB; and grant funding from Janssen, Novartis, Pfizer, and UCB Pharma. SES declares no conflicts of interest relevant to this article.

Address correspondence to Assoc. Prof. P.C. Robinson, University of Queensland School of Clinical Medicine, Royal Brisbane \& Women's Hospital, Herston, Queensland 4006, Australia.

Email:philip.robinson@uq.edu.au.

cross-sectional survey of rheumatologists in Latin America from non-English speaking countries who were affiliated with the Pan American League of Associations for Rheumatology (PANLAR). 5 Their goal was to describe attitudes and practices among rheumatologists during the pandemic. The survey was conducted over 30 days during June and July 2020. At that point, South America was on an upward curve of infection, which we now know would go on to cause devastating rates of infection and death. The study captured responses from 1097 rheumatologists from 19 countries. The majority of responses came from Brazil (25\%), Mexico (21\%), Colombia (13\%), and Argentina (11\%). Respondents were $56 \%$ women and $53 \%$ were under 50 years of age. The majority worked in private practice (82\%), with approximately half also working in institutional outpatient centers and in inpatient care settings.

The authors reported that $7.4 \%$ of respondents had COVID-19, and $9 \%$ of these people were hospitalized. Due to the pandemic, the median hours worked dropped from 27 hours/week to 10 hours/week. Telehealth was used by $79 \%$ of respondents during the pandemic, with phone calls (46\%) and WhatsApp calls (39\%) being the main methods of communication. Of interest, $21 \%$ of respondents did not offer a telehealth option to their patients due to perceived problems with quality of the service and issues with payment. Approximately half restricted themselves to follow-up consultations only (55\%), whereas a minority saw both new and follow-up patients through telehealth (44\%).

The limitations of the study include response bias, as the study was distributed through the national rheumatology association and neither the response rate nor an estimate of it was given. Also, the pandemic would have been in different phases in different areas of Latin America when the survey data were collected. While these are limitations, there were over a thousand responses received from 19 PANLAR countries, so the results likely provide a good insight into the difficulties faced by rheumatologists during the early phase of the pandemic, and could potentially inform the design of measures to better adapt to similar situations in the future.

See Impact of COVID-19 on rheumatology, page 1616 
What does this study tell us about how rheumatologists responded to the pandemic? What does this study tell us about how Latin American rheumatology fared early in the pandemic? First, it is important to acknowledge that Latin America is a heterogenous region with significant social, cultural, political, and economic differences that have led to differences in the response to the COVID-19 pandemic. Latin American health systems are usually composed of a private sector for patients with private insurance, a social security system for salaried workers and their families, and a publicly funded and government-managed health system for low-income patients without access to other sectors. ${ }^{6}$ The pandemic has uniformly presented an extra burden to the already underfinanced and overburdened Latin American public healthcare systems that have been dealing with outbreaks of other vector-borne illnesses. ${ }^{7}$ As expected, a significant decrease in consultations as a result of the pandemic occurred, and this has been reported for the overall practice of outpatient medicine, as well as by rheumatologists in other regions. ${ }^{8}$ In a survey of 858 rheumatologists of the Arab League of Associations of Rheumatology (ArLAR), a decrease in 65\% of outpatient clinics was reported, with similar numbers for both hospitalizations and infusion centers. ${ }^{9}$ This decrease was also associated with a decrease in income. Another survey organized by the COVID-19 African Rheumatology Study Group, with 554 participants from 20 different countries, reported a decrease in face-to-face consultations by $47 \% .{ }^{10}$ Besides shielding measures and national lockdowns, redeployment of rheumatology providers as reported by different studies certainly played a role in these changes. ${ }^{11}$ Outside of clinical care, rheumatologists have also played an active role in research even beyond that involving the treatment of patients with rheumatic disease; they have had important roles in the treatment of several complications of COVID-19. $12,13,14$

The adoption of telemedicine in rheumatology has been quite heterogeneous, and similar to the results reported by Fernández-Ávila and colleagues, ${ }^{5}$ some rheumatologists still have some hesitation about its use even past the pandemic. As reported by the ArLAR survey, telemedicine was adopted by $70 \%$ of participants (mostly telephone and email communications); however, only $22 \%$ of participants confirmed reimbursement of these services. ${ }^{9}$ Although the majority agreed with the use of telemedicine, 1 in 5 participants disagreed. Among participants, lack of a telemedicine platform was reported as the second most important unmet need. Uptake of telemedicine reported by the COVID-19 African Rheumatology Study Group survey utilized telephone (61\%), mobile chats such as WhatsApp (44\%), and emails (16\%) as the most common modalities of telemedicine. ${ }^{10}$ Contrary to what could have been expected, use of telemedicine was not substantially different in developed countries. In a survey distributed among UK rheumatology healthcare professionals including 172 participants (49\% consultants, $25 \%$ nurse specialists, and $12 \%$ specialty trainees), 90\% used this modality in less than 1 in 4 consultations, despite the availability of video consultation for the majority of participants. ${ }^{11}$ The limitations of providing care through telehealth, especially to new patients, are not trivial, but there is also much that can be accomplished in this model of care. Many would agree that for a known patient, the telehealth appointment can provide almost everything except the physical aspects of the patient-doctor interaction. Although rapid implementation and adaptation to telemedicine during the pandemic has not always been a smooth process, hopefully the experience with telemedicine will continue to improve since it often represents a very feasible option. ${ }^{15,16}$

Securing access to care for patients with rheumatic diseases, even more so during the pandemic, is crucial. A recent survey of the Latin American rheumatology workforce by the PANLAR collaborative group showed that on average in Latin America, there is 1 rheumatologist per 106,838 habitants; however, this rate goes as low as 1 per 640,648 habitants in countries like Nicaragua. ${ }^{17}$ Although almost 1 in 4 rheumatologists in Latin America combine a private and public practice, the majority of rheumatologists practice in a private setting, which might further limit the access to patients in a region where there are severe inequalities and a large sector of the population depends on informal unemployment. ${ }^{17,18}$ The shortage in the rheumatology workforce is one of the main barriers of access to care and, as shown by Fernández-Ávila and colleagues, ${ }^{5}$ the ongoing pandemic has most likely worsened this. Although expansion of the Latin American rheumatology workforce is needed to provide high-quality care, the increased use of telemedicine caused by the pandemic may well provide better access and represent an important solution given the concentration of specialists in main cities. ${ }^{17}$

The COVID-19 pandemic has drastically affected the practice of rheumatology in Latin America and beyond, and there is a need to better understand its impact even past the first peaks. Despite the ongoing challenges, the rapid response of healthcare systems and physicians to the pandemic is encouraging and has precipitated changes that will enhance the delivery of care to our patients beyond the pandemic.

\section{REFERENCES}

1. Sparks JA, Wallace ZS, Robinson PC. Coronavirus disease 2019: update on coronavirus disease 2019 outcomes and vaccine efficacy in patients with immune-mediated inflammatory disease. Curr Opin Rheumatol 2021 Jun 23 (Epub ahead of print).

2. Strangfeld A, Schäfer M, Gianfrancesco MA, Lawson-Tovey S, Liew JW, Ljung L, et al. Factors associated with COVID-19related death in people with rheumatic diseases: results from the COVID-19 Global Rheumatology Alliance physician-reported registry. Ann Rheum Dis 2021;80:930-42.

3. Ugarte-Gil MF, Marques CD, Alpizar-Rodriguez D, Pons-Estel GJ, Xibille-Friedmann D, Paiva E, et al. Characteristics associated with Covid-19 in patients with rheumatic disease in Latin America: data from the Covid-19 Global Rheumatology Alliance physician-reported registry. [Internet. Accessed July 14, 2021.] Available from: www.globalrheumpanlar.org/node/254

4. Jacobson SH, Jokela JA. Non-COVID-19 excess deaths by age and gender in the United States during the first three months of the COVID-19 pandemic. Public Health 2020;189:101-3.

5. Fernández-Ávila DG, Barahona-Correa J. Impact of COVID-19 pandemic on rheumatology practice in Latin America. J Rheumatol 2021;48:1616-22.

6. Garcia PJ, Alarcón A, Bayer A, Buss P, Guerra G, Ribeiro H, et al. 
COVID-19 response in Latin America. Am J Trop Med Hyg 2020;103:1765-72.

7. Litewka SG, Heitman E. Latin American healthcare systems in times of pandemic. Dev World Bioeth 2020;20:69-73.

8. Patel SY, Mehrotra A, Huskamp HA, Uscher-Pines L, Ganguli I, Barnett ML. Trends in outpatient care delivery and telemedicine during the COVID-19 pandemic in the US. JAMA Intern Med 2021;181:388-91.

9. Ziadé N, Hmamouchi I, El Kibbi L, Abdulateef N, Halabi H, Abutiban F, et al. The impact of COVID-19 pandemic on rheumatology practice: a cross-sectional multinational study. Clin Rheumatol 2020;39:3205-13.

10. Akintayo RO, Akpabio AA, Kalla AA, Dey D, Migowa AN, Olaosebikan H, et al. The impact of COVID-19 on rheumatology practice across Africa. Rheumatology 2021;60:392-8.

11. Nune A, Iyengar KP, Ahmed A, Bilgrami S, Sapkota HR. Impact of COVID-19 on rheumatology practice in the UK-a pan-regional rheumatology survey. Clin Rheumatol 2021;40:2499-504.

12. Robinson PC, Liew DFL, Liew JW, Monaco C, Richards D, Shivakumar $S$, et al. The potential for repurposing anti-TNF as a therapy for the treatment of COVID-19. Med 2020;1:90-102.
13. Rosas IO, Bräu N, Waters M, Go RC, Hunter BD, Bhagani S, et al. Tocilizumab in hospitalized patients with severe Covid-19 pneumonia. N Engl J Med 2021;384:1503-16.

14. Stone JH, Frigault MJ, Serling-Boyd NJ, Fernandes AD, Harvey L, Foulkes AS, et al. Efficacy of tocilizumab in patients hospitalized with Covid-19. N Engl J Med 2020;383:2333-44.

15. Mortezavi M, Lokineni S, Garg M, Chen YL, Ramsey A. Rheumatology patient satisfaction with telemedicine during the COVID-19 pandemic in the United States. J Patient Exp 2021;8:23743735211008825.

16. Poulsen KA, Millen CM, Lakshman UI, Buttner PG, Roberts LJ. Satisfaction with rural rheumatology telemedicine service. Int J Rheum Dis 2015;18:304-14.

17. Fernández-Ávila DG, Patino-Hernandez D, Kowalskii S, Vargas-Caselles A, Sapag AM, Cachafeiro-Vilar A, et al. Current status of the rheumatologists' workforce in Latin America: a PANLAR collaborative study. Clin Rheumatol 2021;40:2913-20.

18. Intriago M, Maldonado G, Guerrero R, Soriano E, Moreno L, Rios C. LARS study: Latin American rheumatologist survey. Clin Rheumatol 2021;40:377-87. 